VOL. 16 (1977), 36I-369.

\title{
On circulant codes with prescribed distances
}

\section{Deza and Peter Eades}

\begin{abstract}
Necessary and sufficient conditions are given for a square matrix to be the matrix of distances of a circulant code. These conditions are used to obtain some inequalities for cyclic difference sets, and a necessary condition for the existence of circulant weighing matrices.
\end{abstract}

\section{Preliminaries}

Throughout this paper, a circulant code $C$ of length $m$ shall mean a set of $m(0,1)$ codewords of length $m$, with the property that successive codewords differ by a cyclic shift. Thus if $\left(x_{1}, x_{2}, \ldots, x_{m}\right)$ is the first codeword, then $\left(x_{m-j+2}, x_{m-j+3}, \ldots, x_{m-j+1}\right)$ is the $j$ th codeword, for $2 \leq j \leq m$. We can write these codewords as the rows of a circulant $(0,1)$ matrix $X$. The matrix $2(J-X) X^{t}$ is called the matrix of distances of the code $C . \quad(J$ is the $m \times m$ matrix with every entry 1.) Clearly the $(i, j)$ th entry of $2(J-X) X^{t}$ is the (Hamming) distance between the $i$ th and $j$ th codewords. A matrix of the form $2(J-X) X^{t}$, where $X$ is a $(0,1)$ circulant matrix, is called realizable, and the circulant code whose codewords are the rows of $X$ is called the realization of $2(J-X) X^{t}$.

The problem of finding a code with prescribed distances has received attention from both coding theorists and combinatorialists. In Section 2, the realizability of an $m \times m$ matrix is shown to be equivalent to the

Received 5 January 1977. Communicated by Jennifer R. Seberry. 
existence of a partition of $m$ with appropriate properties. Firstly, however, we require some definitions.

Let $x=\left(x_{1}, x_{2}, \ldots, x_{m}\right)$ be a $(0,1)$ codeword. For Sections 1 and 2, we will use the convention that $x_{1}=1$ and $x_{m}=0$. If $x_{i-1} \neq x_{i}=x_{i+1}=\ldots=x_{j} \neq x_{j+1}$, we call $B=(i, i+1, \ldots, j)$ a block of $x$. The blocks of $x$ are numbered $B_{1}, B_{2}, \ldots, B_{t}$ from left to right as they appear in $x$. Note that since $x_{1} \neq x_{m}, t$ is even. Let $X$ denote the circulant with first row $x$, and let $b_{i}$ denote $\left|B_{i}\right|$, the length of the $i$ th block. We say that the sequence $\left(b_{1}, b_{2}, \ldots, b_{t}\right)$ describes $X$.

Note that $m=b_{1}+b_{2}+\ldots+b_{t}$ is an ordered partition of $m$. Consider all the series $b_{j}+b_{j+1}+\ldots+b_{k}$. (If $j>k$, the sum is defined cyclically, that is,

$$
\left.b_{j}+b_{j+1}+\ldots+b_{k}=b_{j}+b_{j+1}+\ldots+b_{t}+b_{1}+\ldots+b_{k} .\right)
$$

For each $1 \leq i \leq[m / 2]$, let $f_{i}$ be the number of such series that have sum $i$ and an odd number of terms, less the number of series that have sum $i$ and an even number of terms. Let $f_{0}$ be $t ;$ then the sequence $\left(f_{i}: 0 \leq i \leq[\mathrm{m} / 2]\right)$ is called the structure of the ordered partition $m=b_{1}+b_{2}+\ldots+b_{t}$.

For example, $(2,3,5,2,1,4)$ describes a circulant code with first codeword (110001llil and blocks $B_{1}=(1,2)$, $B_{2}=(3,4,5), B_{3}=(6,7,8,9,10), B_{4}=(11,12), B_{5}=(13)$, and $B_{6}=(14,15,16,17)$. The structure of $17=2+3+5+2+1+4$ is $(6,1,2,0,1,-1,-1,1,0)$.

\section{The structure theorem}

THEOREM 1 (Structure Theorem). An $m \times m$ matrix $H$ with first row $\left(h_{1}, h_{2}, \ldots, h_{m}\right)$ is realizable if and only if 
(i) $H$ is a symmetric circulant matrix with zero diagonal;

(ii) the entries of $H$ are even, non-negative integers; and

(iii) there is an ordered partition $m=b_{1}+b_{2}+\ldots+b_{t}$ with stmucture $\left(f_{i}\right)$ satisfying $f_{0}=h_{2}$ and $f_{i}=\frac{z}{2}\left(2 h_{i+1}-h_{i}-h_{i+2}\right)$ for $1 \leq i \leq[\mathrm{m} / 2]$.

Proof. Suppose $H$ is realized by a circulant code whose codewords are the rows of a circulant $(0, I)$ matrix $X$. Let $\left(b_{1}, b_{2}, \ldots, b_{t}\right)$ denote the sequence that describes the first row of $X$, and let $\left(f_{i}\right)$ be the structure of $m=b_{1}+b_{2}+\ldots+b_{t}$. Clearly $(i)$ and (ii) follow, and $h_{2}=f_{0}$. Now

$$
h_{i}=2 \sum_{q=1}^{m}\left(x_{q}-x_{q}^{x} q+i-1\right),
$$

and we can deduce

$$
\frac{1}{2}\left(2 h_{i+1}-h_{i}-h_{i+2}\right)=\sum_{q=1}^{m} t_{i q}
$$

where $t_{i q}=\left(x_{q}-x_{q-1}\right)\left(x_{q+i-1}-x_{q+i}\right)$.

But $t_{i q}$ is non-zero only when $x_{q} \neq x_{q-1}$ and $x_{q+i-1} \neq x_{q+i}$. In this case, $q$ must be the first element of some block $B_{k}$, and $q+i$ must be the last element of some block $B_{q}$. Hence if $t_{i q} \neq 0$, then $b_{k}+b_{k+1}+\ldots+b_{l}=i$. But $t_{i q}=1$ if $2-k+1$ is odd, and $t_{i q}=-1$ if $l-k+1$ is even. So $f_{i}=\frac{1}{2}\left(2 h_{i+1}-h_{i}-h_{i+2}\right)$, for $i \geq 1$.

Conversely, suppose that $H$ is a symetric circulant of even nonnegative integers, and suppose the partition $m=b_{1}+b_{2}+\ldots+b_{t}$ has structure $\left(f_{i}\right)$ satisfying $f_{0}=h_{2}$ and $f_{i}=\frac{\hbar}{2}\left(2 h_{i+1}-h_{i}-h_{i+2}\right)$, for $i \geq 1$. We claim that $H$ is the matrix of distances of a circulant code with first codeword described by $\left(b_{1}, b_{2}, \ldots, b_{t}\right)$. Let $x$ be the $(0,1)$ circulant with first row described by $\left(b_{1}, b_{2}, \ldots, b_{t}\right)$. If 
$\left(h_{1}^{\prime}, h_{2}^{\prime}, \ldots, h_{m}^{\prime}\right)$ is the first row of $2(J-X) X^{t}$, then by the argument above,

$$
h_{2}=h_{2}^{\prime}=f_{0},
$$

and

$$
2 h_{i+1}-h_{i}-h_{i+2}=2 f_{i}=2 h_{i+1}^{i}-h_{i}^{\prime}-h_{i+2}^{\prime} \text { for } i \geq 1 \text {. }
$$

Since $h_{1}^{\prime}=h_{1}=0$, these equations suffice to ensure that $h_{i}=h_{i}^{\prime}$ for $1 \leq i \leq m$.

\section{Applications}

From the structure theorem, a cyclic difference set (see [1]) with parameters $(v, k, \lambda)$ exists if and only if there is an ordered partition of $v$ with structure $\left(f_{i}\right)$ satisfying $f_{0}=2(k-\lambda), f_{1}=k-\lambda$, and $f_{i}=0$ for $2 \leq i \leq[v / 2]$. We can prove some inequalities for partitions with these properties.

PROPOSITION 2. Suppose there is an ondered partition $v=b_{1}+b_{2}+\ldots+b_{t}$ with stmucture $\left(f_{i}\right)$ satisfying $f_{0}=2 n$, $f_{1}=n$, and $f_{2}=f_{3}=0$. Let $p_{i}$ be the number of times that $i$ occurs in $\left(b_{1}, b_{2}, \ldots, b_{t}\right)$. Then, whenever $v>11 n / 3$ and $n \geq 4$,

$$
\begin{gathered}
p_{1}=\frac{1}{2} t=n, \\
\max (0,4 n+1-v, n-[(v-n) / 4]) \leq p_{2} \leq[3 n / 4],
\end{gathered}
$$

and

$$
\max \left(0,5 n-v-2 p_{2}\right) \leq p_{3} \leq \min \left(n-p_{2}-1,2 p_{2}, 3 n-4 p_{2}\right)
$$

Proof. By definition, $f_{0}=2 n=t$; and $f_{1}$ is the number of ones in $\left\{b_{1}, b_{2}, \ldots, b_{t}\right\}$, hence we have (1).

A one-run $A$ in the sequence $b=\left(b_{1}, b_{2}, \ldots, b_{t}\right)$ is a subsequence $A=\left(b_{i}, b_{i+1}, \ldots, b_{j}\right)$ where $b_{i}=1=b_{i+1}=\ldots=b_{j}$. Of course, we define one-runs cyclically: if $j<i$, then 


$$
A=\left(b_{i}, b_{i+1}, \ldots, b_{t}, b_{1}, \ldots, b_{j}\right) \text {. }
$$

Let $u$ be the number of one-runs of $b$, and let $w$ be the number of oneruns with precisely one entry. Then the number of times $(1,1)$ occurs in $b$ is $\sum(|A|-1)$, where the sum runs over all the one-runs $A$ of $b$. But

$$
\begin{aligned}
\sum(|A|-1) & =\left(\sum|A|\right)-u \\
& =p_{1}-u \\
& =n-u .
\end{aligned}
$$

Thus, since $f_{2}=0$, we obtain

$$
p_{2}=n-u \text {. }
$$

Now the number of times that $(1,1,1)$ occurs in $b$ is $\sum(|A|-2)$, where the sum runs over all one-runs of $b$ with more than one entry. But

$$
\begin{aligned}
\sum(|A|-2) & =(n-w)-2(u-v) \\
& =2 p_{2}+w-n .
\end{aligned}
$$

\section{Hence}

$$
w \geq \max \left\{0, n-2 p_{2}\right\}
$$

Now let $x_{1}$ and $x_{2}$ be the number of times $(1,2)$ and $(2,1)$ occur in $b$ respectively. Then, for $i=1,2, x_{i} \leq u$ and $x_{i} \leq p_{2}$. Hence

$$
\begin{aligned}
x_{1}+x_{2} & \leq 2 \min \left\{u, p_{2}\right\} \\
& =2 \min \left\{n-p_{2}, p_{2}\right\} .
\end{aligned}
$$

Using $f_{3}=0$ and (5), we obtain

$$
p_{3}+\left(2 p_{2}-n+w\right) \leq 2 \min \left\{n-p_{2}, p_{2}\right\}
$$

This, together with (6) implies 
(8)

$$
\begin{aligned}
p_{3} & \leq 2 \min \left\{n-p_{2}, p_{2}\right\}+n-2 p_{2}-\max \left\{0, n-2 p_{2}\right\} \\
& =\min \left\{3 n-4 p_{2}, 2 p_{2}\right\} .
\end{aligned}
$$

Let $C=\left\{b_{i}: b_{i} \neq 1,2\right.$, or 3$\}$. Since $p_{1}=n=\frac{5}{2} t$, $|c|=n-p_{2}-p_{3}$. We show that $|c|>0$. For if $|c|=0$, then $n-p_{2}=p_{3} ;$ so by (8), $n-p_{2} \leq z_{2}$, so $p_{2} \geq n / 3$. But $v=p_{1}+2 p_{2}+3 p_{3}=4 n-p_{2} ;$ hence $p_{2}=4 n-v$. But this implies $4 n-v \geq n / 3$, and thus $v \leq 11 n / 3$, contrary to our assumptions.

Hence $|c|=n-p_{2}-p_{3} \geq 1$. So

$$
p_{3} \leq n-p_{2}-1
$$

Also, $\sum_{c \in C} c \geq 4|c|$, since $c \geq 4$ for all $c \in C$. This gives $v-p_{1}+2 p_{2}+3 p_{3} \geq 4 n-p_{2}-p_{3}$, and by (1) we have

$$
p_{3} \geq 5 n-2 p_{2}-v
$$

Now (8), (9), and (10) together give (3). From (3) we obtain $\max (0,4 n+1-v,(5 n-v) / 4) \leq p_{2} \leq[3 n / 4]$. But since $n \geq 4$, this is the same as (2). This completes the proof of Proposition 2.

Using Baumert's list of known difference sets [1], one can check that the inequalities (2) and (3) are sharp; that is, for each inequality in (2) and (3), there is a difference set for which equality holds.

A circulant Hadamard matrix is a circulant $(1,-1)$ matrix whose rows are mutually orthogonal. Konvalina and Kosloski [3] have defined a circulant quasi-Hadamard matrix to be a circulant $(-1,1)$ matrix whose first row is orthogonal to all but possibly one of the succeeding rows. Let $p_{i}$ be the number of blocks of length $i$ in the first row of a circulant quasi-Hadamard matrix of order $4 n \geq 16$. Konvalina and Kosloski noted that $P_{1}=n$; from Proposition 2 we can also deduce

$$
n / 4 \leq p_{2} \leq 3 n / 4
$$

and 


$$
\max \left\{0, n-2 p_{2}\right\} \leq p_{3} \leq \min \left\{n-p_{2}-1,2 p_{2}, 3 n-4 p_{2}\right\}
$$

Since a circulant Hadamard matrix is a circulant quasi-Hadamard matrix, this gives bounds on the length of blocks in the first row of a circulant Hadamard matrix.

We can also calculate $p_{i}$ for partitions with more general structure.

PROPOSITION 3. Let $p_{i}$ be the number of times that $i$ occurs in the ordered partition $m=b_{1}+b_{2}+\ldots+b_{t}$. For $i \geq 2$, let

$$
m_{i}=m-\sum_{j=1}^{i-1} j p_{j}
$$

and

$$
t_{i}=t-\sum_{j=1}^{i-1} p_{j} .
$$

Then $m_{i} \geq i t_{i}$, and

(i) if $m_{i}=i t_{i}$, then $p_{i}=t_{i}$; and

(ii) if $m_{i}>$ it $t_{i}$, then $p_{i} \leq t_{i}-1$.

Proof. Note that $t_{i}$ is the number of summands $b_{j}$ which are greater than or equal to $i ; m_{i}$ is their sum. Thus $m_{i} \geq i t_{i}$ is immediate, and if $m_{i}=i t_{i}$, then each $b_{j}$ which is greater than $i-1$ must be $i$; thus $p_{i}=t_{i}$. And if $m_{i}>i t_{i}$, then $p_{i}<t_{i}$; that is, $p_{i} \leq t_{i}-1$

Finally, the structure theorem gives a necessary condition for the existence of circulant weighing matrices. An $m \times m(0, \pm 1)$ matrix satisfying $W W^{t}=k I_{m}$ is called a weighing matrix of weight $k$ and order $m$. The problem of determining for which $k$ and $m$ a circulant weighing matrix of weight $k$ and order $m$ exists is discussed in [2].

Suppose $X$ and $Y$ are $(0,1)$ circulants described by $b=\left(b_{1}, b_{2}, \ldots, b_{n}\right)$ and $c=\left(c_{1}, c_{2}, \ldots, c_{u}\right)$ respectively. Let 
$x=\left(x_{1}, x_{2}, \ldots, x_{m}\right)$ and $y=\left(y_{1}, y_{2}, \ldots, y_{m}\right)$ be the first rows of $x$ and $y$ respectively. We will assume that $x_{1}=1$ and $x_{m}=0$, but we will allow $y_{1}=y_{m}$. If $X$ and $Y$ are disjoint, that is, $x_{i}=1$ implies $y_{i} \neq 1$, then we say the sum of $b$ and $c$ is the sequence which describes $X+Y$.

COROLLARY 4. Suppose there is a circulant weighing matrix $W$ of weight $k$ and order $m$. Then there are partitions $m=b_{1}+b_{2}+\ldots+b_{n}$ and $m=c_{1}+c_{2}+\ldots+c_{u}$ with sum $m=d_{1}+d_{2}+\ldots+d_{v}$ with the following properties:

$$
\begin{aligned}
& \text { (i) } b_{1}+b_{3}+\ldots+b_{n-1}=(k+\sqrt{k}) / 2 ; \\
& \text { (ii) } c_{2}+c_{4}+\ldots+c_{u}=(k-\sqrt{k}) / 2 ; \\
& \text { (iii) } d_{1}+d_{3}+\ldots+d_{v}=k ;
\end{aligned}
$$

and if $\left(f_{i}\right),\left(g_{i}\right)$, and $\left(h_{i}\right)$ are the structures of the partitions $m=b_{1}+b_{2}+\ldots+b_{n}, m=c_{1}+c_{2}+\ldots+c_{u}$, and $m=d_{1}+d_{2}+\ldots+d_{v}$, respectively, then

$$
\begin{aligned}
& \text { (iv) } 2 f_{0}+2 g_{0}-h_{0}=2 k ; \\
& \text { (v) } 2 f_{1}+2 g_{1}-h_{1}=k ; \\
& \text { (vi) } 2 f_{i}+2 g_{i}-h_{i}=0 \text {, for } i \geq 2 \text {. }
\end{aligned}
$$

Proof. Write $W$ as $X-Y$, where $X$ and $Y$ are $(0,1)$ circulants. Since $W W^{t}=2 X X^{t}+2 Y Y^{t}-(X+Y)(X+Y)^{t}$, the corollary follows from the structure theorem.

\section{References}

[1] Leonard D. Baumert, Cyclic difference sets (Lecture Notes in Mathematics, 182. Springer-Verlag, Berlin, Heidelberg, New York, 1971). 
[2] Peter Eades and Richard M. Hain, "On circulant weighing matrices", Ars Combinatoria (to appear).

[3] John Konvalina and Rodney H. Kosloski, "Cyclic quasi-Hadamard matrices", Utilitas Math. (to appear).

Centre National des Recherches Scientifiques, Université de Paris VII,

Paris,

France;

Department of Pure Mathematics, School of General Studies,

Australian National University,

Canberra, ACT. 